\title{
Une anomalie fonctionnelle : le proglissement
}

\section{RÉSUMÉ}

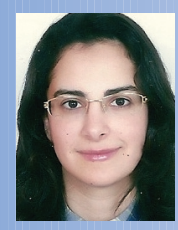

Salwa REGRAGUI

Professeur agrégé en orthodontie,

Chef du département

d'orthopédie dento-faciale

Faculté de médecine dentaire de Rabat,

Certificat d'études supérieures

en Biomatériaux utilisés en art dentaire,

Certificat d'études supérieures

en orthopédie dento-faciale,

Département d'Orthopédie Dento-Faciale,

Faculté de Médecine Dentaire de Rabat,

BP 6212, Rabat Instituts. Rabat. Maroc.

\section{Fatima ZAOUI}

Professeur de l'enseignement supérieur au Service d'orthopédie dento-faciale, Faculté de médecine Dentaire de Rabat.
Le proglissement est une anomalie fonctionnelle se traduisant par un articulé inversé antérieur et des rapports dentaires latéraux de classe III en raison d'une position avancée de la mandibule.

Cet aspect clinique est assez inquiétant pour les parents et peut faire suspecter à tort une dysmorphose de classe III.

Le proglissement est une urgence thérapeutique puisque, non traitée, cette anomalie fonctionnelle plutôt simple risque de se transformer, avec la croissance, en vraie classe III squelettique.

Une fois le diagnostic du proglissement posé, sa correction par la levée des obstacles occlusaux est simple et a des répercussions spectaculaires sur l'esthétique faciale par le rétablissement d'une harmonie squelettique et musculaire. 


\section{Introduction}

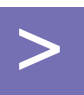

Le proglissement est une anomalie fonctionnelle dont le signe le plus marquant est l'inversé de l'articulé antérieur incisif. Cette occlusion croisée antérieure est associée à des rapports de classe III canine et molaire, ou encore à un articulé inversé bilatéral (fig. $1 \mathrm{a}, 1 \mathrm{~b}$ ).

Ces rapports occlusaux s'expliquent par une position avancée de la mandibule qui s'établit

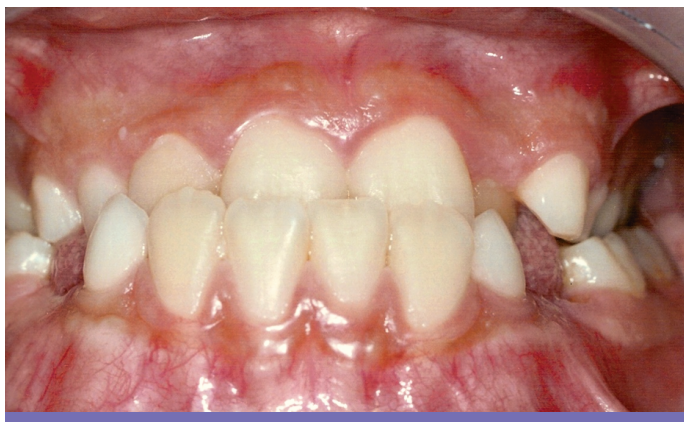

Fig. 1 a Articulé inversé antérieur.

\section{Diagnostic}

La nécessité d'un diagnostic précoce s'impose de par la responsabilité du proglissement dans la genèse d'éventuels problèmes parodontaux (récessions gingivales sur les incisives inférieures vestibulo-versées), dans la perturbation de la phonation et de l'esthétique faciale "visage boudeur», et surtout dans l'évolution d'un problème fonctionnel simple en problème squelettique dont le traitement est plus compliqué.

Dans le proglissement, la fonction incisale n'existe plus. Or, selon la théorie de Delaire [4] lors de la recherche par le sujet d'une position d'occlusion confortable.

En effet, des canines lactéales trop pointues, l'éruption des incisives centrales en palatoversion ou celle des incisives inférieures en vestibuloversion génèrent des prématurités dentaires ne pouvant être évitées que par un avancement de la mandibule [1, 2, 3]. Le proglissement est une urgence thérapeutique qui doit être diagnostiquée tôt.

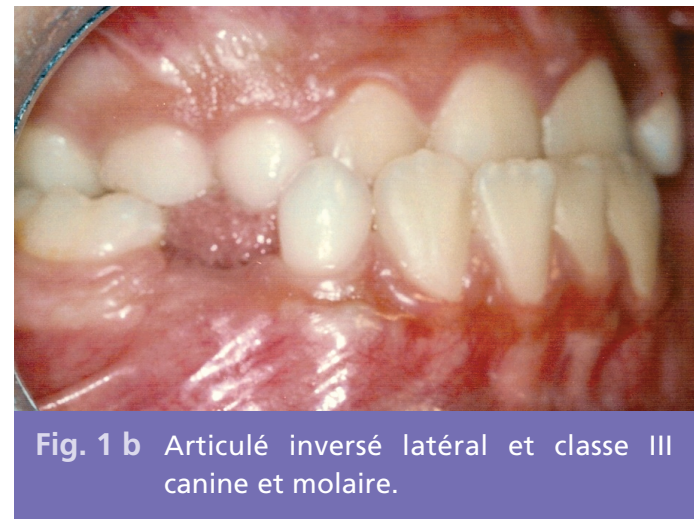

c'est la proprioception incisive (mise en jeu dès que la denture temporaire devient fonctionnelle) qui conditionne le développement du maxillaire.

Ces forces occlusales stimulent la croissance de l'os frontal auquel elles sont transmises à travers les apophyses montantes du maxillaire; dans son mouvement vers l'avant, la corticale externe du frontal entraîne avec elle le maxillaire.

De là, toute diminution précoce des forces masticatoires incisivo-canines est responsable 


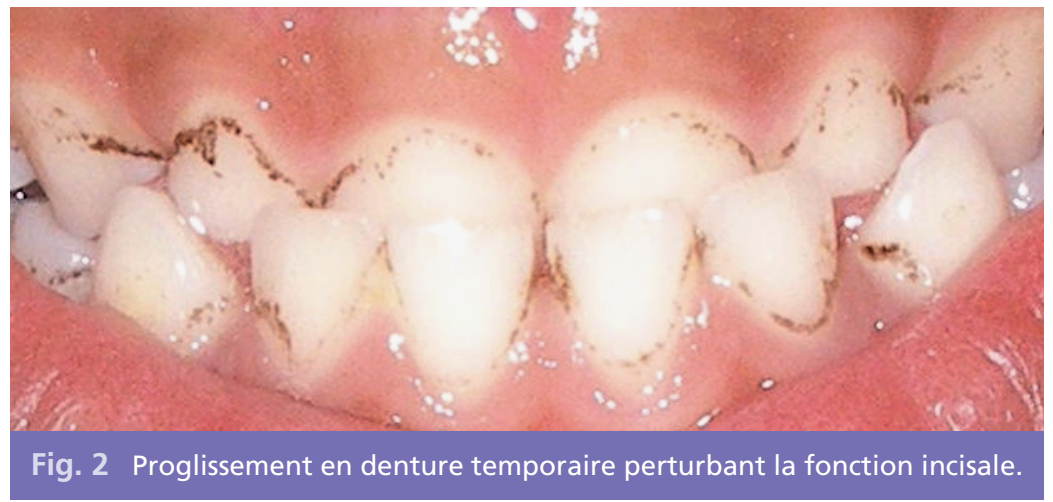

d'un hypodéveloppement du frontal et par conséquent du maxillaire (fig. 2).

Par ailleurs, la croissance mandibulaire est stimulée puisque la clé incisive ne joue pas son rôle de maintien d'une croissance mandibulaire équilibrée.
Ainsi s'installe progressivement une classe III squelettique par défaut de croissance maxillaire associée à un excès de développement mandibulaire.

\section{Pourquoi faire un diagnostic différentiel entre proglissement et classe III ?}

L'aspect de l'articulé antérieur inversé est assez inquiétant pour les parents qui, dans ces cas, suspectent à tort un problème squelettique de classe III.

Pour que le praticien puisse rassurer les parents et leur affirmer que le problème est dentaire et non squelettique, que son traitement est simple et ne nécessite pas un traitement orthopédique par activation de la croissance du maxillaire et freinage de celle de la mandibule, ou encore un traitement orthodontico-chirurgical à la fin de la croissance, il faut qu'il fasse un diagnostic différentiel entre un proglissement et une vraie classe III.

Ce diagnostic différentiel repose sur :

- la manœuvre de Nevrezé $[5,6]$;
- l'examen clinique exo- et endobuccal ;

- l'examen de la téléradiographie de profil.

La manœuvre de Nevrezé consiste à guider la mandibule pour recentrer les condyles dans leur cavités glénoïdes. Elle comprend trois temps :

- 1er temps : abaissement de la mandibule pour échapper aux blocages occlusaux ;

$-2^{\mathrm{e}}$ temps : rétropulsion de la mandibule guidée par l'opérateur vers le haut et l'arrière, bouche ouverte ;

$-3^{e}$ temps : fermeture en relation centrée.

La manœuvre de Nevrezé est considérée comme positive si l'on obtient au moins le bout à bout incisif (fig. 3 a, 3 b). 


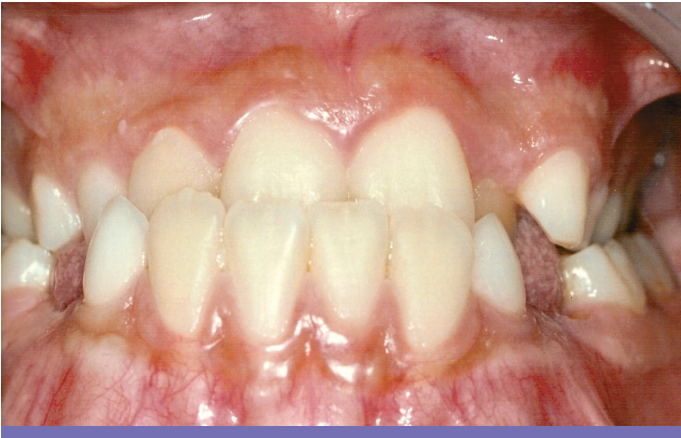

Fig. 3 a Articulé inversé antérieur.

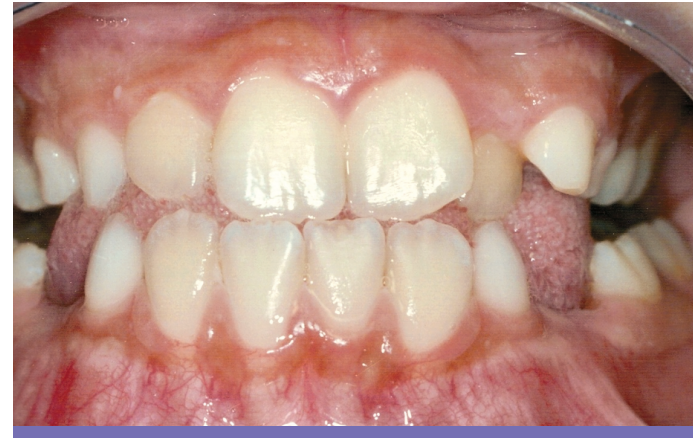

Fig. 3 b Bout à bout incisif = manœuvre de Nevrezé positive $\rightarrow$ proglissement.

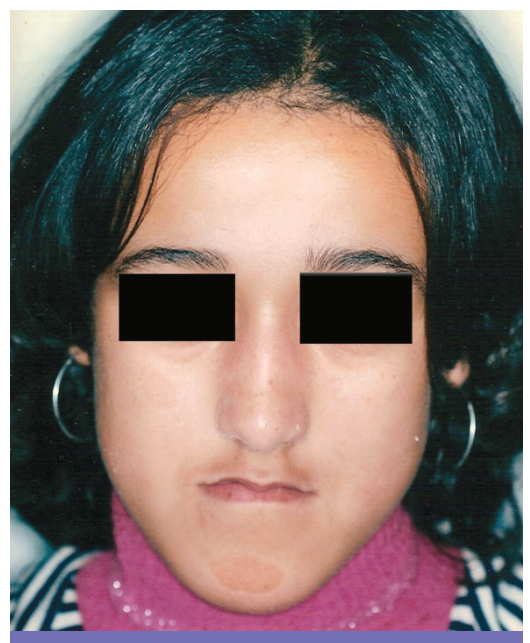

Fig 4 a Visage caractéristique d'une classe III squelettique.

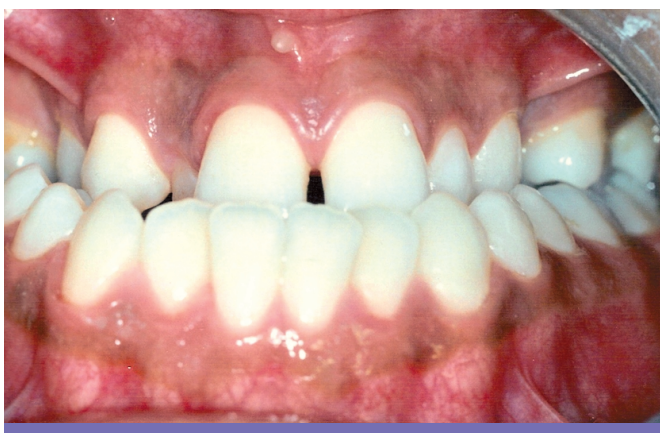

Fig. 4 b Compensations incisives de classe III contraires aux inclinaisons des axes des incisives supérieures et inférieures dans le proglissement.
Dans les cas où l'inversé de l'articulé est en rapport avec une classe III squelettique, il y a impossibilité de reculer la mandibule puisque les condyles sont centrés dans leurs cavités glénoïdes.

Dans les cas où la classe III squelettique est associée au proglissement, la manœuvre de
Nevrezé réduit l'inversé de l'articulé sans arriver jusqu'au bout à bout incisif.

Par ailleurs, I'examen clinique exo- et endobuccal révèle (fig. 4 a, 4 b) :

- l'absence des caractéristiques maxillo-faciales propres aux classes III (pommettes effacées, sillons nasogéniens marqués révélateurs d'un 
petit maxillaire, mandibule et menton bien développés...) ;

- l'absence des compensations incisives de classe III (incisive inférieure linguo-versée et incisive supérieure vestibulo-versée) en faveur d'une incisive inférieure droite ou en proalvéolie et d'une incisive supérieure palatoversée.

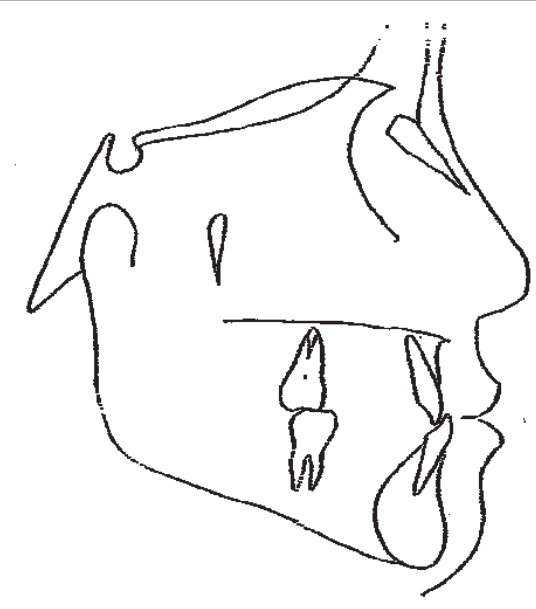

Fig. 5 a Tracé céphalométrique en occlusion de convenance (proglissement).

\section{Traitement}

La thérapeutique consiste à éliminer la cause du proglissement et à coordonner les arcades.

La mandibule se recentre spontanément et l'occlusion normale où la clé incisive est ainsi obtenue s'oppose à la récidive du proglissement.

Si la cause du proglissement est une prématurité au niveau des canines temporaires, il faut en meuler la pointe [7].
Le $3^{e}$ élément du diagnostic est la prise de la téléradiographie de profil en occlusion de convenance et en relation centrée : le proglissement est confirmé par la normalisation des rapports entre les bases maxillaire et mandibulaire sur le cliché en relation centrée (fig. 5 a, 5 b).

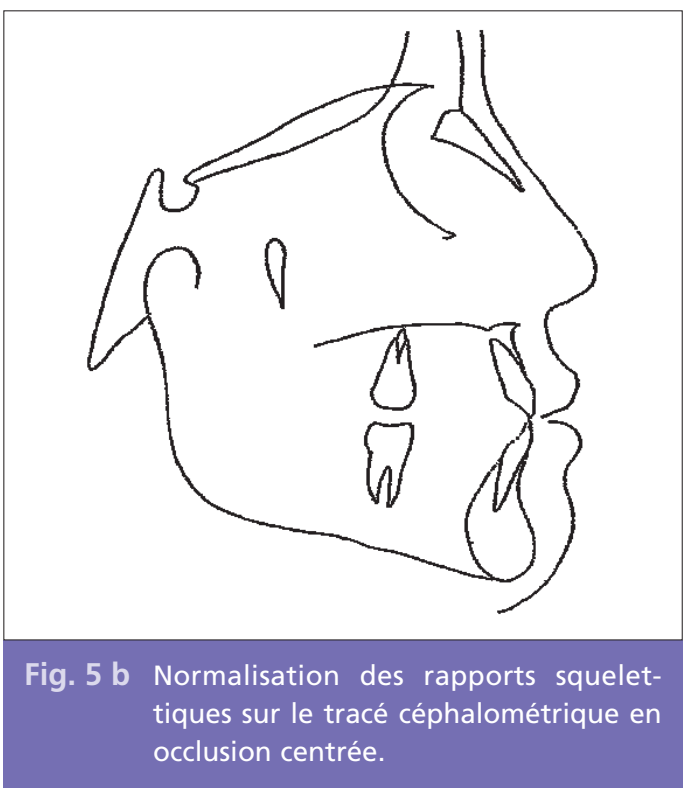

Si la cause du proglissement est une palatoversion des incisives maxillaires permanentes, l'expansion maxillaire sera obtenue par une thérapeutique orthodontique amovible [8] ou fixe.

Quelle que soit la technique adoptée, une surélévation de l'occlusion est souhaitable pour échapper au blocage occlusal antérieur. 


\section{Cas cliniques}

Cas clinique $\mathrm{n}^{\circ} 1$ : thérapeutique amovible

(fig. 6 a, 6 b, 6 c).

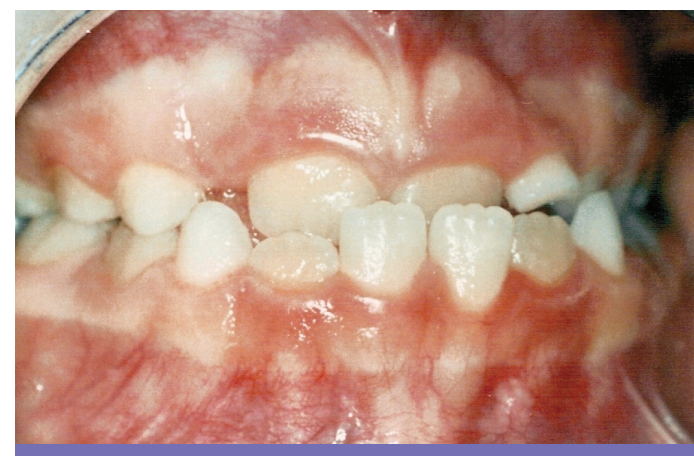

Fig. 6 a Patiente âgée de 9 ans présentant un proglissement en rapport avec des incisives supérieures palatoversées.

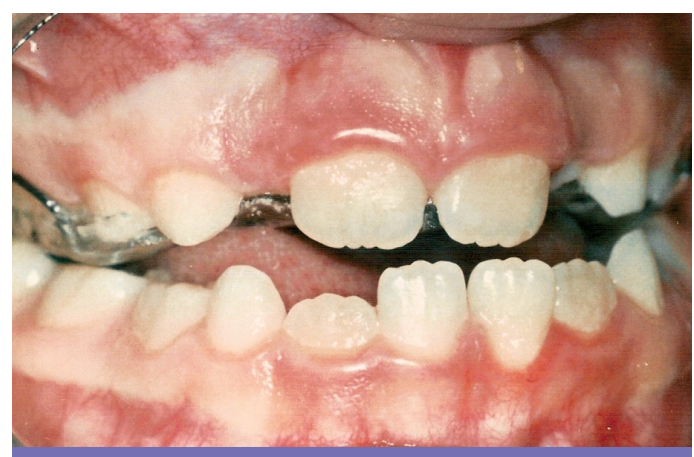

Fig. 6 b Vestibulisation des incisives par une plaque à vérin à action postéro-antérieure dont le vérin est activé 2 fois par semaine et dont l'épaisseur de la résine réalise la surélévation de l'articulé.

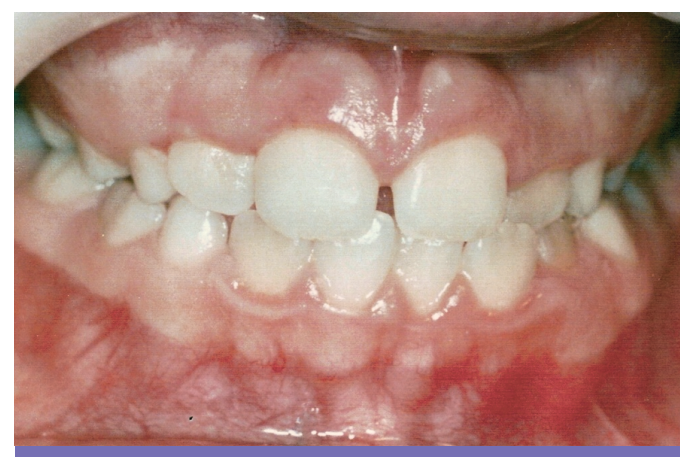

Fig. 6 c Rétablissement de l'articulé en 2 mois, puis surveillance jusqu'à l'établissement de la denture définitive. 
Cas clinique $\mathrm{n}^{\circ} 2$ : thérapeutique fixe

(fig. 7 a, 7 b, 7 c, 7 d).
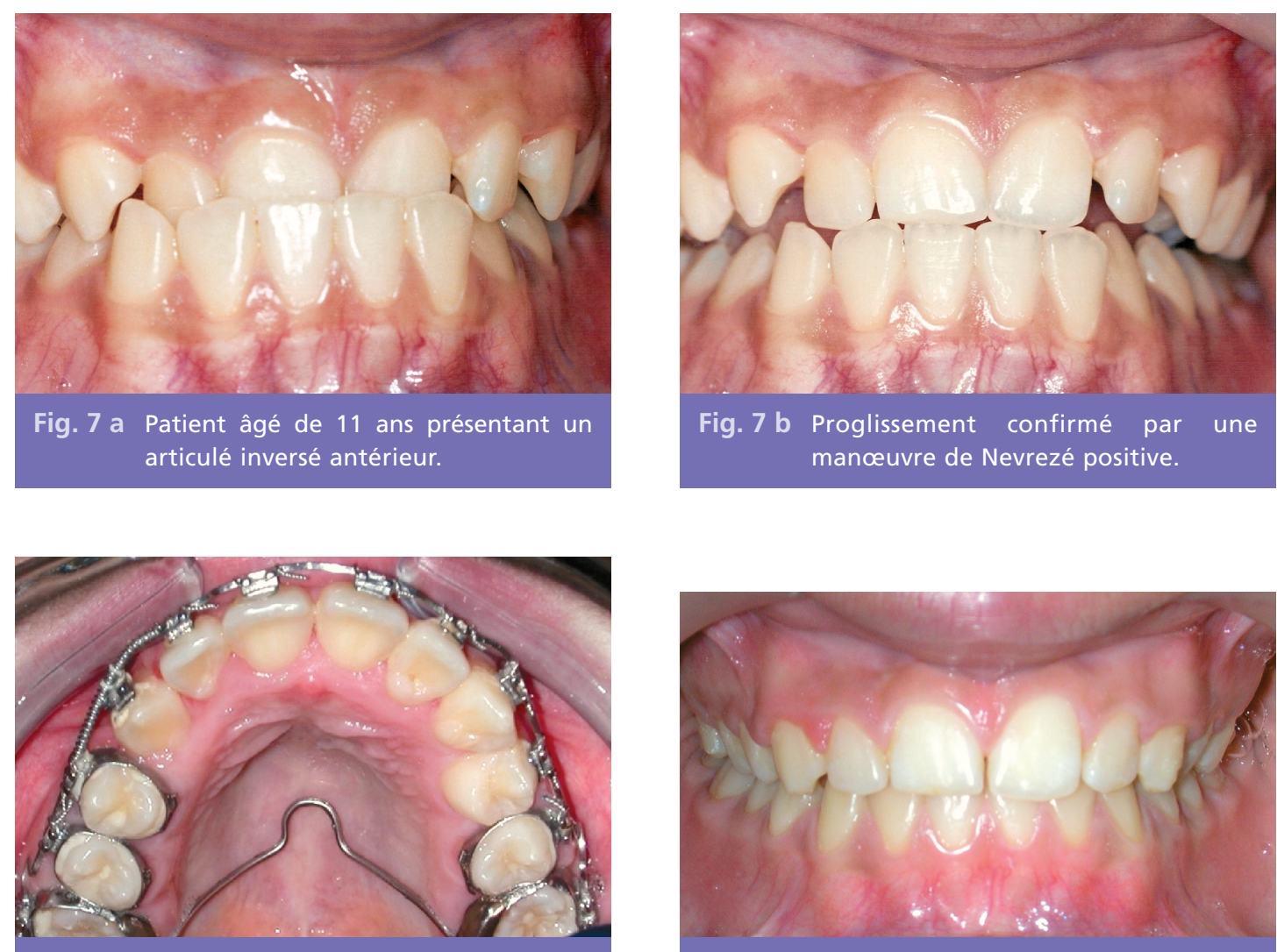

Fig. 7 c Collage d'un dispositif multi-attaches maxillaire permettant la vestibulisation des incisives après surélévation de l'occlusion par des cales en verre-ionomères placées sur les faces occlusales des 1 res molaires inférieures.

\section{Conclusion}

Le proglissement est une urgence thérapeutique en ODF puisque, non traitée, cette anomalie fonctionnelle plutôt bénigne risque de se transformer, avec la croissance, en vraie dysmorphose de classe III.

Le traitement du proglissement est dentoalvéolaire, contrairement aux classes III où I'on doit agir sur les bases osseuses; d'où l'importance de faire un diagnostic différentiel entre les deux.

Ainsi, un traitement précoce est souhaité visant à améliorer l'esthétique et surtout la fonction et à éviter les problèmes parodontaux. 


\section{Bibliographie}

1. Fellus $\mathrm{P}$.

Orthodontie précoce

en denture

temporaire.

Paris : Éditions CdP, 2003.

2. Patti A, Perrier d'Arc G.

Les traitements

orthodontiques

précoces.

Paris : Quintessence

International, 2003.

3. Rakosi T, Irmtrud J.

Atlas

de médecine

dentaire.

Orthopédie

dento-faciale.
Diagnostic.

Paris : Flammarion, 1992.

4. Delaire J.

Le concept cortical.

International Orthod

2006;4(3).

5. Rotenberg $\mathrm{M}$, Lodter $\mathrm{C}$.

L'orthodontie

des malocclusions

de classe III

par proglissement.

Rev Orthop Dento Faciale

1997;31:79-88.

6. Le Gall M, Philip C, Bandon D.

Arch Pediatr

2009;16(1):77-83.
7. Soulet A.

Suppression

des causes

de déviation

de la croissance.

Rev Orthop Dento Faciale

1996;30:445-459.

8. Isaacson KG, Muir JD,

Reed RT.

Removable

orthodontic

appliances.

Paris :

Éditions Elsevier

Science, 2002.

A functional anormaly:

mandibular forward shift

Salwa REGRAGUI,

Fatima ZAOUI

\section{Keywords \\ - mandibular forward shift \\ - dysfonction \\ - class III \\ - diagnosis \\ - therapeutic}

Mandibular forward shift is a functional anormaly being translated by an anterior crossbite and of the side dental reports of class III because of a advanced position of the mandible.

This clinical aspect is worrying enough for the parents and can make suspecter wrongly a dysmorphose of class III.

Mandibular forward shift is an emergency therapeutic since, untreated, this anormaly rather simple functional calculus is likely to change, with the growth, in true class III skeletal.

Once the diagnosis of mandibular forward shift posed, its correction by the lifting of the occlusal obstacles is simple and has spectacular effects on facial esthetics by the re-establishment of a skeletal and muscular harmony. 


\section{Questions - Réponses}

1/ Réponse par vrai ou faux

Le proglissement est une fausse classe III

$$
\text { Vrai } \square \quad \text { Faux }
$$

2/ QCM à une seule réponse

Le proglissement :

a - est une anomalie squelettique

b - est causé par un problème dentaire

c - nécessite un traitement orthopédique

c - est une anomalie dans laquelle la mandibule est trop en arrière

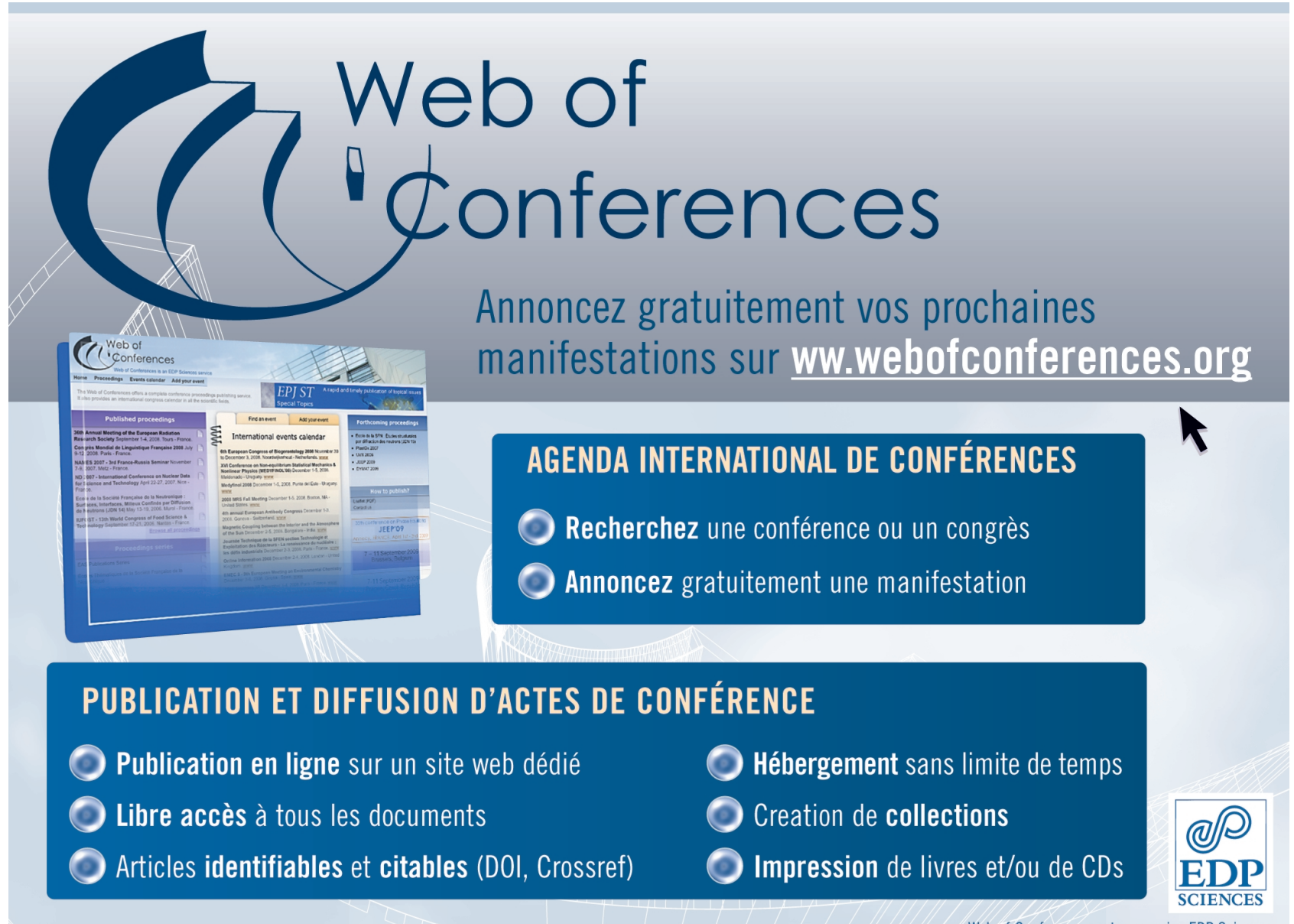


Pour s'abonner, composer le :

tél. + 33 (0)1 $69181514-\mathrm{fax}+33(0) 169860678$

France et Union Européenne

version papier + version électronique $199 €$

Étudiant* : France et Union Européenne

(* sur présentation d'une attestation)

version papier + version électronique $119 €$

Reste du monde

version papier + version électronique $229 €$

Membre de l'AEOS

$179 €$

Prix au numéro

$65 €$

Frais d'envoi par avion

$49 €$

\section{NUMÉROS(*)}

(*) Offre valable jusqu'au 31/10/09

Réglé la somme de

le

Chèque bancaire / postal $\mathrm{N}^{\circ}$

ou carte $\mathrm{N}^{\circ}$

(Partie à conserver)

(Partie à joindre à tout règlement)

$\square$ Je souhaite m'abonner pour

à la Revue AOS

$\square$ Je souhaite prolonger mon abonnement pour

à la Revue AOS

\section{MODE DE PAIEMENT :}

$\square$ Paiement effectué par chèque à l'ordre de : EDP Sciences - REVUE AOS

17 avenue du Hoggar - P.A. de Courtabœuf

B.P. 112 - 91944 Les Ulis Cedex A (France)

Tél. + 33 (0)169181514 - Fax + 33 (0)1 69860678

e-mail : abonnements@edpsciences.org

$\square$ Paiement par carte bancaire : $\square$ Carte Visa

Master Card

$\mathrm{N}^{\circ}$

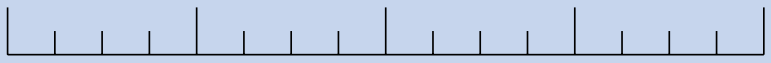

Noter ici les 3 derniers chiffres situés au dos de la carte

Date d'expiration $\lfloor+|1|$

Signature obligatoire

\section{VOTRE ADRESSE :}

Vous êtes: $\square$ Chirurgien-Dentiste $\square$ Stomatologue $\square$ Orthodontiste $\square$ Autre: précisez

Nom :

Prénom :

Adresse :

Code Postal

Ville

Tél

Fax

E-mail 